\title{
Unusual Presentation of Foreign Body in a Pediatric Patient
}

\author{
Manjit Talwar, Meenakshi Awana
}

\begin{abstract}
Missed foreign bodies are one of the leading causes of malpractice claims made againstemergency physicians. Foreign bodies in the lip are not common. Here, a case of a child presenting with tooth fragment embedded in the lower lip after a fall is being reported. Accurate diagnosis was made by palpation and radiographic evaluation. The foreign body was removed surgically under local anesthesia with prompt recovery and esthetics was preserved. The patient was taken up for root canal treatment. It is essential to look for foreign bodies embedded in soft tissue particularly in children after a fall, which if overlooked can have significant complications.
\end{abstract}

Keywords: Foreign body, Apexification, Swelling, Esthetics, Misdiagnosis.

How to cite this article: Talwar M, Awana M. Unusual Presentation of Foreign Body in a Pediatric Patient. Int J Experiment Dent Sci 2012;1(2):110-112.

\section{Source of support $\mathrm{Nil}$}

Conflict of interest: None

\section{INTRODUCTION}

Detection and removal of foreign bodies may cause anxiety and frustration for the physician with significant clinical implications. Inability to detect a foreign body can lead to its retention in the soft tissues with resultant complications. For the patient, a retained foreign body can mean significant morbidity in the form of infection, pain, multiple visits, high cost and extensive surgery. $M$ issed foreign bodies are one of the leading causes of malpractice claims made against emergency physicians. ${ }^{1}$ Foreign bodies in the lip are not common. The object may be superficial, deep or displaced. ${ }^{2}$ Inadvertently swallowed foreign objects enter the gastrointestinal tract approximately $92.5 \%$ of the times and the tracheobronchial tree in $7.5 \%$ of these instances. ${ }^{3}$ Here, a case of a tooth fragment embedded in the lower lip of a 9 -year-old child is being reported.

\section{CASE REPORT}

A 9-year-old boy reported to the Department of Dentistry at Government M edical College and Hospital, Sector 32, Chandigarh, India, with history of a fall. His main concern was the fracture of his upper front teeth were not esthetic (Fig. 1). On clinical examination fracture of incisal edges of 11, 21 was observed with pulp involvement (Ellis class III \#). Swelling of the upper and lower lips was observed (Fig. 2). Initial examination did not show any foreign body in the oral cavity or oropharynx. He had no breathing or respiratory difficulty. $V$ itals were normal. On palpation of

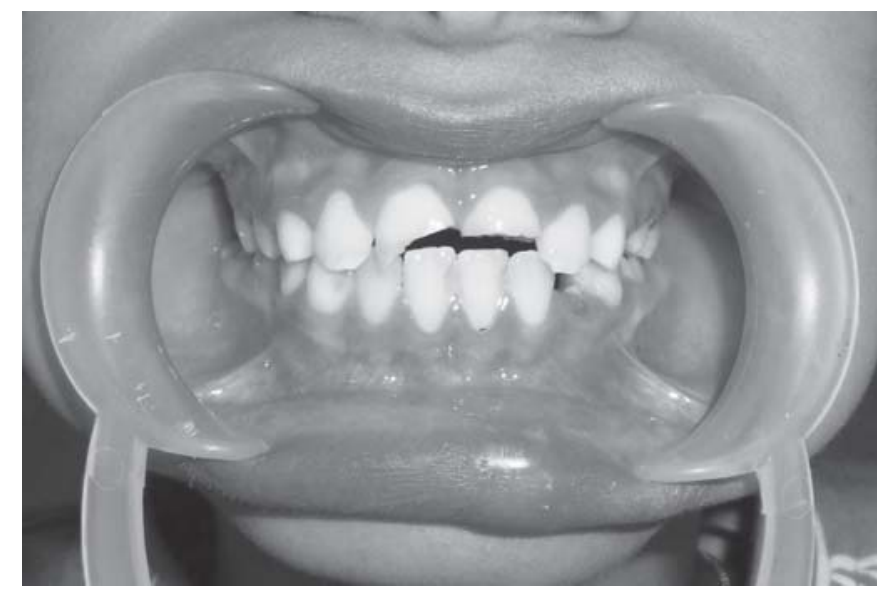

Fig. 1: Fractured maxillary central incisors with exposed pulp

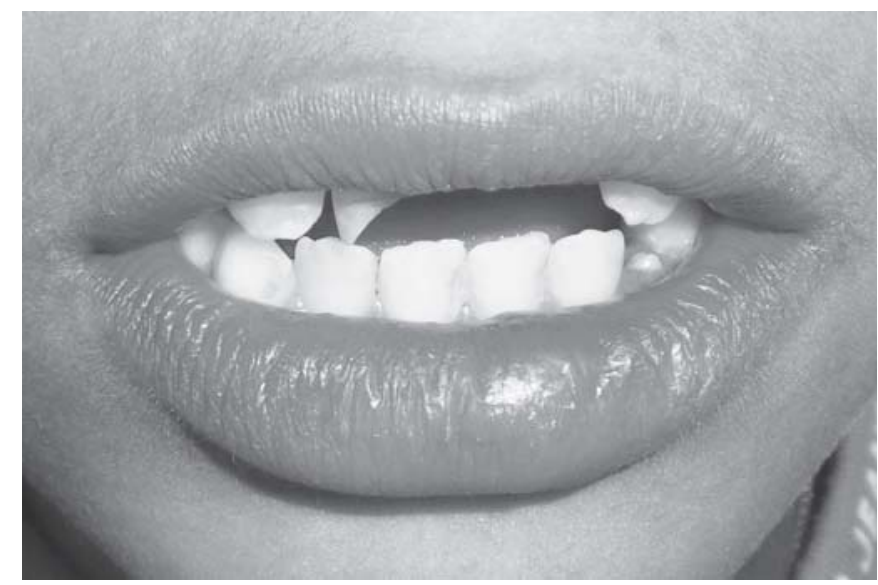

Fig. 2: Swelling of the lower lip

the lower lip, a firmness was observed that was not normal and appeared to be other than fibrotic. Radiographic investigation was done for the lower lip which revealed a radiopaque object of about $0.5 \times 0.5 \mathrm{~cm}$ (Fig. 3). R oot canal opening was done to relieve the patient of pain.

A ntibiotics and anal gesics were prescribed. Patient was asked to report after 1 week. The foreign body was removed surgically under local anesthesia (Fig. 4) and sutures given, that were removed after 1 week. The radiographic object was the fractured fragment of the upper left central incisor which got embedded in the lower lip due to impact of the fall and the lip had significantly healed. Composite build up was done for 11, 21 (Fig. 5). A pexification was carried out as the apical closure had not occurred which normally takes place 4.5 years after eruption of the central incisors (i.e. for central incisors which erupts at 7 years, root formation is completed by 11 years). As the child was 9 years, only 2.5 years had elapsed since eruption. This was followed by obturation of the maxillary central incisors (Fig. 6). 


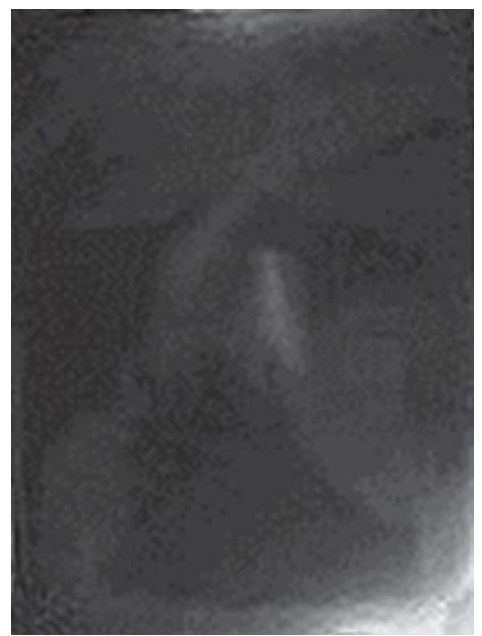

Fig. 3: Radiograph of lower lip showing foreign body

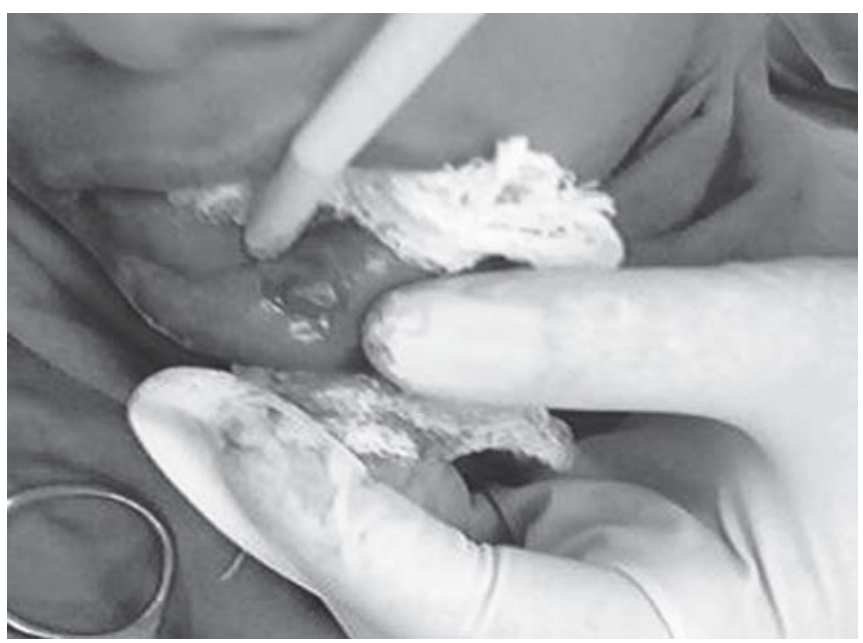

Fig. 4: Surgical removal of the foreign body

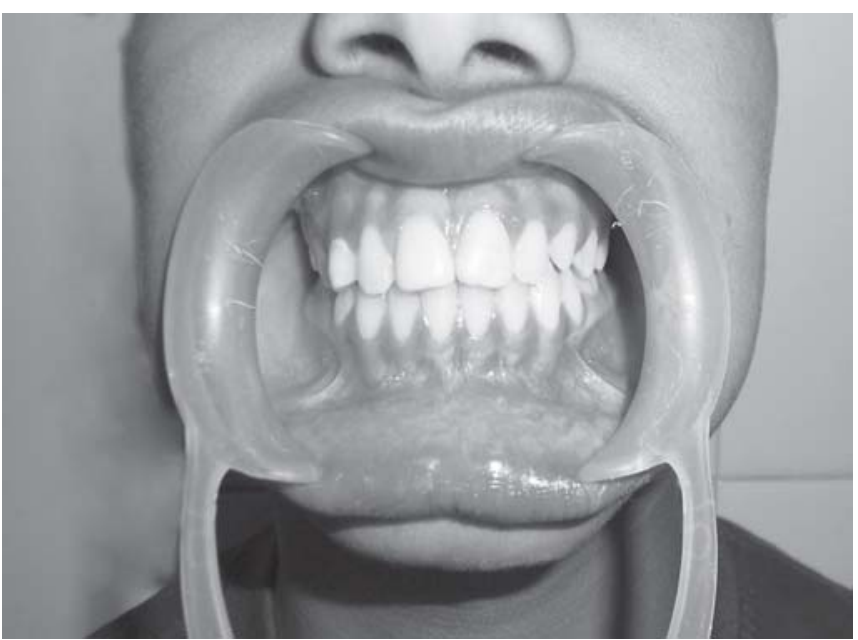

Fig. 5: Composite build-up of maxillary central incisors

\section{DISCUSSION}

Tooth avulsion in people who sustain multiple injuries is common and can have significant complications if not recognized. Trauma-related penetration of teeth into the

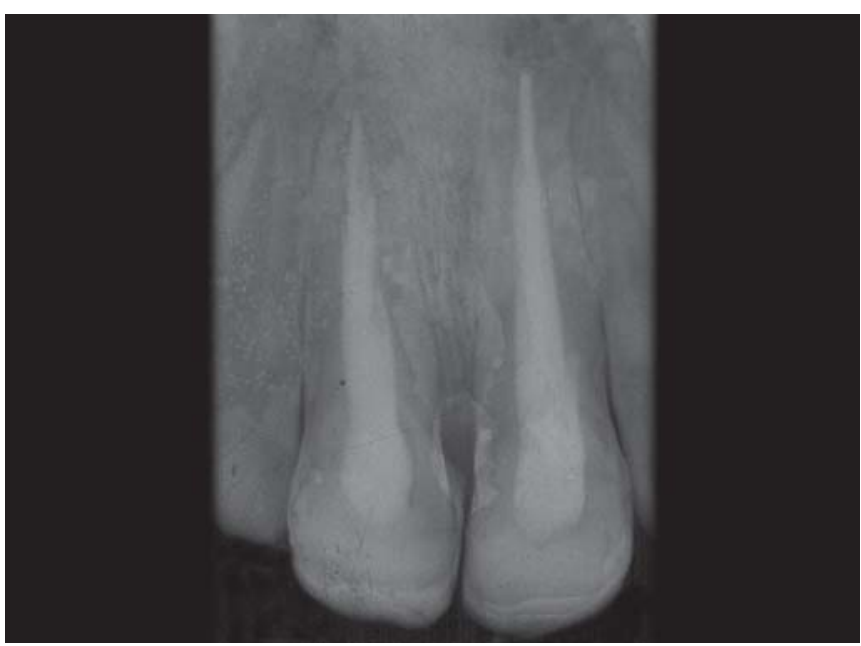

Fig. 6: Radiograph showing obturation of maxillary central incisors after apexification

maxillary sinus, frontal sinus, pyriform sinus and soft tissues of the head and neck has been described. If loose, a tooth in the oral cavity can either be sw allow ed or aspirated. ${ }^{4}$ These can result in decrease in the gag reflex, swallowing incoordination or any other impairment of the protective airway mechanism. ${ }^{3}$ There are innumerable instances of foreign bodies getting embedded into the maxillofacial tissues in the emergency surgical practice following facial trauma. In such situations, oral and maxillofacial surgeons are frequently facing situations requiring quick decisions in dental offices or even in hospitals where advanced imaging technologies, like computed tomography (CT) or magnetic resonance imaging (M RI) are not available. In this situation, it is advisable to use conventional radiographic techniques in order to correctly diagnosis and to locate the foreign body. Even if the exact position is known from imaging data, the accurate reproduction of its position in the patient's body may be difficult if the foreign body is not adjacent to a definitive anatomical landmark. The major determinant of injury is behavior of penetrating object within the tissue which in turn depends on deformation, yaw (rotation about the long axis) and fragmentation of the projectile. The foreign body removal can be delayed in approximately one-third of all foreign bodies, because they are initially radiographically missed or misdiagnosed. ${ }^{5}$ In the present case proper clinical examination and radiographic evaluation was the landmark of the diagnosis and treatment. Taking into consideration the child's age, the surgical procedure was conducted very conservatively so that esthetics could be preserved.

\section{CONCLUSION}

While performing oral examination subsequent to a fall especially in children, it is essential to look for pieces of teeth, pencils, debris, etc. embedded in soft tissue, which 
may be overlooked with considerable implications for occurrence of complications. ${ }^{6}$

\section{REFERENCES}

1. Graham DD J r. UItrasound in the emergency department: Detection of wooden foreign bodies in the soft tissues. J Emerg M ed 2002 J an;22(1):75-79.

2. da Silva EJ, Deng $Y$, Tumushime-Buturo CG. A n unusual foreign body in the tongue. $\mathrm{Br}$ J Oral Maxillofac Surg 2000;38(3):241-42.

3. Fields RT Jr, Schow SR. A spiration and ingestion of foreign bodies in oral and maxillofacial surgery: A review of the literature and report of five cases. J Oral Maxillofac Surg 1998;56(9):1091-98.

4. Mahmood S, Lello GE. Tooth in the nasopharynx. Br J Oral M axillofac Surg 2002;40:448-49.
5. Dhadhich A, Jaju P. R etrieval of unusual foreign body from the lip. J Oral Health Res 2010;3:97-99.

6. Bhardwaj PK. Tooth fragment embedded in upper lip: A diagnostic consideration. Clin Dent 2010;5:16-18.

\section{ABOUT THE AUTHORS}

\section{Manjit Talwar}

A ssociate Professor, Department of D entistry, Government M edical College and Hospital, Chandigarh, India

\section{Meenakshi Awana (Corresponding Author)}

Senior Resident, Department of Dentistry, Government M edical College and Hospital, Sec-32, Chandigarh, India, e-mail: minaxiawana@gmail.com 